\title{
The Effect of Intracameral Adrenaline Bolus Injection on Pupil Size, Pulse Rate and Blood Pressure During Small Incision Cataract Surgery
}

\section{Sonal Muley ${ }^{1}$, Chetan Saoji ${ }^{2}$, Sachin Daigavane ${ }^{3}$}

\begin{abstract}
'Senior Resident, Department of Ophthalmology, Datta Meghe Medical College, Shalinitai Meghe Hospital \& Research centre, (Datta Meghe Institute of Medical Sciences) Hingana Nagpur-441110; ${ }^{2}$ Assistant Professor, Department of Ophthalmology, Datta Meghe Medical College, Shalinitai Meghe Hospital \& Research centre, (Datta Meghe Institute of Medical Sciences) Hingana Nagpur-441110; 3Professor and HOD, Department of Ophthalmology, Jawaharlal Nehru Medical College, Datta Meghe Institute of Medical Sciences, Wardha-442001.
\end{abstract}

\section{ABSTRACT}

Aim: Pupillary miosis during cataract surgery and irrigation and aspiration $(\mathrm{I} / \mathrm{A})$ is found to be a major cause of iris trauma, cortex retention, posterior capsule rent, vitreous loss, and even posterior nucleus dislocation. Adequate mydriasis is necessary for safe cataract surgery. Intracameral adrenaline bolus injection can help maintain mydriasis. We designed a prospective interventional study to assess the efficacy and safety of intracameral adrenaline bolus injection after nucleus delivery, as an adjunctive to preoperative topical mydriatics.

Methods: Forty patients with cataract were divided into two groups: Study group and the control group. The study group (20 patients) received intracameral bolus preservative-free adrenaline injection in the dose of 0.1 cc of 1:5000 concentration after nucleus delivery. The diameter of the pupil was measured by surgical calipers before and after nucleus delivery, after injection of intracameral adrenaline in the study group and after IOL placement in both the groups. Pulse rate, systolic, and diastolic pressure were measured before and after the cataract surgery.

Results: The mean pupil size in the study group after nucleus delivery was $5.33 \mathrm{~mm}$ and $5.32 \mathrm{~mm}$ in the control group. A significant pupillary dilatation was observed in the study group 30 seconds after the injection of bolus dose of intracameral adrenaline. The mydriasis obtained in the study group (mean $7.67 \mathrm{~mm}$ ) was significantly greater than the control group mean $(p<0.0001)$.

The mean pupil size after IOL dialing was $6.86 \mathrm{~mm}$ in the study group which was significantly greater than the control group(mean $4.20 \mathrm{~mm}$ ). The mydriasis maintained in the study group was significantly greater than the control group ( $p<0.0001)$. Pulse rate and blood pressure showed no significant changes during the surgery in both the groups.

Conclusion: We concluded that intracameral preservative-free adrenaline bolus injection ( $0.1 \mathrm{cc}$ of $1: 5000)$ is a safe and effective adjunctive to topical mydriatics in maintaining mydriasis during surgery.

Key Words: SICS, Intracameral, Adrenaline, Bolus, Cataract, Dilatation, Mydriasis

\section{INTRODUCTION}

Cataract surgery is performed better if dilatation of the pupil is maintained until the intraocular lens (IOL) has been inserted $^{1}$. Failure to maintain the dilatation during surgery can escalate the risk of iris injury, cortex retention, or the more dreadful complication, posterior capsule rent ${ }^{2}$. Adrenaline must be tried before mechanical pupil dilation methods (iris hooks, sphincterotomy) because these methods have some drawbacks, including iris damage and sphincter rupture. Mechanical dilation is also expensive, time-consuming and may require more incisions ${ }^{3}$.
The pupil size is dependent on the net effect of opposing factors: adrenergic sympathetic activity stimulating the dilator pupillae thereby causing dilatation ${ }^{4}$; and cholinergic parasympathetic activity acting on sphincter pupillae with the induction of pupillary constriction. Epinephrine directly stimulates the dilator pupillae, but when applied topically to the eye, the 1:1000 solution does not infiltrate into the eye in adequate concentration to produce mydriasis 5 .

Preoperative dilatation in cataract surgery is normally achieved by a topical adrenergic agonist with good penetration of the cornea, such as phenylephrine $5 \%$ and an anticho-

\section{Corresponding Author:}

Dr. Sonal Muley, Senior Resident Department of Ophthalmology, Datta Meghe Medical College, Shalinitai Meghe Hospital \& Research Centre, (Datta Meghe Institute of Medical Sciences) Hingana Nagpur-441110; Mob: 9890959395; Email: Ambad.sawan@gmail.com

ISSN: 2231-2196 (Print)

Received: 03.06 .2020
ISSN: 0975-5241 (Online)

Revised: 26.06 .2020
Accepted: 08.07.2020
Published: 21.07 .2020 
linergic drug such as tropicamide $0.8 \%$. During surgery, there is a tendency of the pupil to shrink, particularly after iris manipulation and nucleus delivery ${ }^{6}$. Adrenaline can be directly injected into the anterior chamber intracamerally to help achieve adequate mydriasis of the pupil for more safe and effective surgery.

\section{MATERIALS AND METHODS}

This prospective interventional study was carried out in Ophthalmology Department at Datta Meghe Medical College, Shalinitai Meghe Hospital and Research Centre, Nagpur in collaboration with Jawaharlal Nehru Medical College, Datta Meghe Institute of Medical Sciences, Sawangi (Meghe) Wardha, Maharashtra.

Forty cases (25 women, 15 men) undergoing routine extracapsular cataract extraction was randomly divided into two groups- study group and control group. The study group received intracameral adrenaline bolus injection at the dose of $0.1 \mathrm{cc}$ of 1:5000 concentration. The control group did not receive intracameral adrenaline.

All patients underwent full ophthalmic and general physical examination including measurement of blood pressure and pulse rate, random blood sugar and electrocardiogram. Fasting blood sugar, post-prandial sugar, and $\mathrm{HbAlc}$ was recorded in diabetic patients. All patients gave informed consent before inclusion in the study.

\section{Inclusion criteria}

Patients of either sex undergoing routine cataract surgery.

\section{Exclusion criteria}

1. Known allergy to any of the drugs.

2. Previous intraocular inflammation.

3. Patients with traumatic cataract.

4. Patients with congenital cataract

5. Patients with glaucoma

6. Patients with corneal opacities or degenerations
All patients received four drops of phenylephrine 5\% and tropicamide $0.8 \%$ during the one-hour preceding surgery. All patients in both groups were administered peribulbar block anaesthesia employing a solution of $1: 1$ compounded $2 \%$ lidocaine plus adrenaline 1:200,000 (Lox 2\%, Neon Laboratories, India) and $0.5 \%$ bupivacaine (Anawin $0.25 \%$, Neon Laboratories, India), with hyaluronidase (Hynidase, Shreya Life Sciences Pvt. Ltd., India) added at 7.5 turbidity units $/ \mathrm{ml}$ to the solution.

All patients were operated by manual small incision cataract surgery by a single surgeon and by the same technique

20 eyes (Study group) received intracameral bolus injection of preservative free adrenaline (in the dosage of $0.1 \mathrm{cc}$ of $1: 5000)$ after the nucleus delivery. $1 \mathrm{ml}$ of $1 \mathrm{mg} / \mathrm{ml}(1: 1000)$ adrenaline (Vasocon, Neon Laboratories Ltd., India) was diluted with $4 \mathrm{ml}$ of ringer lactate solution. $0.1 \mathrm{ml}$ of this solution (adrenaline 1:5000) was used to inject intracamerally.

20 eyes (control group) did not receive any intracameral adrenaline injection.

Pupil diameters were measured by surgical calipers before and after nucleus delivery (before injection of intracameral adrenaline, 30 seconds after injection of adrenalin and after IOL dialing in the bag.

Each patient's pulse rate and systolic and diastolic pressure were recorded before and after the surgery.

Data Analysis: Data was analyzed using an un-paired student ' $\mathrm{t}$ ' test.

\section{OBSERVATION AND RESULTS}

The present study included 40 Cases (25 females and 15 males). The mean age of the patients was 64.57 years (SD 7.86).

14 patients had a history of type 2 diabetes mellitus. Of these, intracameral adrenaline was injected in 8 patients.

Table 1: Pupil size changes between study and control groups:

\begin{tabular}{lcccc} 
Groups & $\begin{array}{c}\text { Before Nucleus } \\
\text { Delivery } \\
(\mathbf{M m})\end{array}$ & $\begin{array}{c}\text { After Nucleus Delivery (Before } \\
\text { Adrenaline Injection In Study } \\
\text { Group)(Mm) }\end{array}$ & $\begin{array}{c}\text { After Adrenaline } \\
\text { Injection In Study } \\
\text { Group(Before I/A)(Mm) }\end{array}$ & $\begin{array}{c}\text { After Iol } \\
\text { Insertion(Mm) }\end{array}$ \\
Study Group & $7.77 \pm 0.67$ & $5.33 \pm 0.35$ & $7.67 \pm 0.29$ & $6.86 \pm 0.37$ \\
Control Group & $7.99 \pm 0.56$ & $5.32 \pm 0.36$ & $5.32 \pm 0.36$ & $4.205 \pm 0.32$ \\
P-value & 0.266 & 0.92 & $<0.0001$ & $<0.0001$ \\
\hline
\end{tabular}


Table No 1 shows Control groups showed a progressive shrinkage of the pupil from $7.99 \pm 0.56 \mathrm{~mm}$ before nucleus delivery to $5.32 \pm 0.36 \mathrm{~mm}$ after nucleus delivery and $4.205 \pm 0.32 \mathrm{~mm}$ after IOL dialing.

The study group showed an initial pupil constriction after nucleus delivery, the pupil was re-dilated using intracameral adrenaline, the dilatation achieved after injection in the study group was significant ( $p$-value $<0.0001$ ) in comparison to the control group. The mydriasis maintained after IOL dialing in the study group was significantly greater as compared to control group ( $\mathrm{p}$-value $<0.0001)$

Table 2: Pulse rate changes before and after surgery in study and control groups:

\begin{tabular}{lccc} 
Groups & $\begin{array}{c}\text { Before Surgery } \\
(/ \mathrm{min})\end{array}$ & $\begin{array}{c}\text { After Surgery } \\
(/ \mathrm{min})\end{array}$ & P value \\
Study Group & $79.2 \pm 5.78$ & $79.27 \pm 4.50$ & 0.76 \\
Control Group & $80.7 \pm 6.23$ & $80.5 \pm 7.30$ & 0.92 \\
\hline
\end{tabular}

Table no 2 shows: The pulse rate changes before and after surgery in both study group ( $p$ value 0.76 ) and control group ( $p$ value 0.92 ) was not significant.
Table 3: Systolic BP changes before and after surgery in study and control groups:

\begin{tabular}{llll} 
Groups & Before Surgery & After Surgery & P value \\
Study group & $128.7 \pm 9.67$ & $128.2 \pm 7.59$ & 0.85 \\
Control group & $125.8 \pm 7.89$ & $125.7 \pm 8.99$ & 0.97 \\
\hline
\end{tabular}

Table no 3 shows: The systolic pressure changes before and after surgery in both study group ( $p$ value 0.85 ) and control group ( $p$ value 0.97 ) was not significant.

Table 4: Diastolic BP changes before and after surgery in study and control groups:

\begin{tabular}{lccc} 
Group & Before Surgery & After Surgery & P-value \\
Study Group & $78.8 \pm 5.89$ & $81.6 \pm 4.56$ & 0.1 \\
Control Group & $79.6 \pm 6.41$ & $81.3 \pm 5.55$ & 0.37 \\
\hline
\end{tabular}

Table no 4 shows: The diastolic pressure changes before and after surgery in both study group (p value 0.1 ) and control group ( $p$ value 0.37 ) was not significant.

\section{Table 5: Pupil size changes in diabetic patients.}

\begin{tabular}{lcccc} 
Groups & $\begin{array}{c}\text { Before Nucleus } \\
\text { Delivery } \\
(\mathbf{M M})\end{array}$ & $\begin{array}{c}\text { After Nucleus Delivery (Be- } \\
\text { fore Adrenaline Injection In } \\
\text { Study Group)(MM) }\end{array}$ & $\begin{array}{c}\text { After Adrenaline Injection } \\
\text { In Study Group(Before I/A) } \\
(\mathbf{M m})\end{array}$ & $\begin{array}{c}\text { After Iol } \\
\text { Insertion(Mm) }\end{array}$ \\
\hline Study Group & $7.45 \pm 0.60$ & $4.25 \pm 0.23$ & $7.12 \pm 0.29$ & $6.22 \pm 0.23$ \\
Control Group & $8 \pm 0.40$ & $4.55 \pm 0.26$ & $4.55 \pm 0.26$ & $4.1 \pm 0.13$ \\
P Value & 0.07 & 1.0 & $<0.0001$ & $<0.0001$ \\
\hline
\end{tabular}

14 patients had a history of type 2 diabetes mellitus. Of these, intracameral adrenaline was injected in 8 patients.

Table no. 5 shows that significant pupillary dilatation achieved with injection of intracameral adrenaline in diabetic patients $(7.45 \pm 0.60)$ which was maintained till IOL insertion( $6.22 \pm 0.23)$ in comparison to control groups $(\mathrm{p}<0.0001)$.

\section{DISCUSSION}

Manual small-incision cataract surgery (MSICS or SICS) is a low-cost, small-incision type of extracapsular cataract extraction (ECCE) that is mainly in use in the developing countries?.

The mean age of the patients in our study was 64 years. Old age and frailty is found to be associated with cataract ${ }^{8}$ and other systemic co-morbidities? .

One particular disadvantage in SICS is the constriction of the pupil just after the nucleus delivery (especially if nucleus delivery has been done with the use of wire vectis or phacosandwich method or irrigating wire vectis) which makes cortical clean up a uphill task which may lead to iris injury, prolonged surgical time or the dreaded complication of posterior capsular rupture ${ }^{10}$.

14 patients in our study had a history of type 2 diabetes mellitus. Of these, intracameral adrenaline was used in 8 patients. Diabetes mellitus is associated with increased difficulty in cataract surgery due to poor pupillary dilatation, the tendency of the pupil to constrict during surgery, difficult hydrodissection ${ }^{11-14}$. Diabetes mellitus and raised $\mathrm{HbAlc}$ is also associated with diabetic retinopathy ${ }^{15}$. No significant complications occurred during this surgery. The mean pupillary dilatation achieved in diabetic patients after injection of intracameral adrenaline was $7.45 \mathrm{~mm}$ and the dilatation was maintained till IOL insertion $(6.22 \mathrm{~mm})$. Hence, intracameral adrenaline is useful in the maintenance of mydriasis in diabetic patients in addition to topical mydriatics for safe cataract surgery. This finding is supported by other studies ${ }^{16}$. 
Supplemental adrenaline bolus injection can be used intracamerally in lower concentrations just after the nucleus delivery to maintain mydriasis until the implantation of an intraocular lens. Preservative free adrenaline was used in this study in the dosage of $0.1 \mathrm{cc}$ of 1:5000.

Reports of corneal endothelial damage and cystoid macular edema have been reported with higher concentrations of adrenaline $(1: 1000)^{17,18}$. Adrenaline is a known risk factor for pseudophakic cystoid macular edema, but intracameral adrenaline at a concentration of $0.2 \mathrm{mg} / \mathrm{mL}$ or lesser (1:5000) has been shown as not to be associated with increased risk of cystoid macular edema ${ }^{17}$. There have been a few reports of severe corneal decompensation after the use of the adrenaline in 1: 1000 concentration $^{18}$ but not 1: $5000^{19}$. Hull et al. showed that the corneal endothelial damage was a result of toxicity due to the sodium bisulphite preservative in the adrenaline preparation and not the adrenaline itself ${ }^{19,20}$.

Therefore, using preservative-free low concentration bolus injection of intracameral adrenaline at a dose of $0.1 \mathrm{cc}$ of 1:5000 offers a definite advantage in SICS.

Also, some authors suggest the use of adrenaline infusion with I/A containing adrenaline in the concentration of $1: 1000,000^{21,22}$. This method, however, has some drawbacks like contact of anterior segment for a long time with adrenaline and its preservative and also less control over the total volume of adrenaline entering the eye.

The present study indicates that there is pupillary miosis immediately after nucleus delivery in both the groups after supplementing bolus adrenaline injection, there is immediate pupillary dilatation in the study group which is maintained till the end of the surgery. The control group shows a significantly smaller pupil size till the end of surgery $(p$ value $<0.0001)$. No significant complications were recorded in the present study.

Our results were comparable to the study conducted by Liou, et al. which compared the bolus injection of $0.1 \mathrm{cc}$ intracameral adrenaline in 5 different concentrations 1:25000, $1: 50000,1: 100,000,1: 200,000$, and 1:400,000 during phacoemulsification. The 1:400,000 concentrations was shown to be as effective as 1:25,000, but the cases of the 1:400,000 group failed to maintain the mydriasis after irrigation and aspiration. The mean pupillary dilatation in all the five groups was significantly greater than the control group ${ }^{23}$.

One particular concern is the changes in pulse rate and blood pressure in patients with the use of intracameral adrenaline. No significant changes in pulse rate or blood pressure were noted in the present study. This finding is supported by previous studies ${ }^{21-24}$.

A study by Hassan et al. showed that adrenaline even in higher concentrations was safe on pulse blood pressure. No significant changes were observed in cardiovascular, cornea, or macula in their study ${ }^{24}$.

Therefore, in manual small incision cataract surgery, use of adrenaline is safe and effective means of redilating the pupil as well as maintaining the pupil size.

Our study suffered from a few shortcomings notably the non-randomized nature of study which makes drawing any definite conclusion impossible. Also, our sample size in the present study was very small. But this study underscores the need for the design of a larger randomized control study to study the question at hand.

\section{CONCLUSION}

We concluded that the use of intracameral preservative-free adrenaline bolus injection in lower concentrations could be a safe and effective adjunctive to topical mydriatics in manual small incision cataract surgery.

Acknowledgement: Authors acknowledge the immense help received from the scholars whose articles are cited and included in references of this manuscript. The authors are also grateful to authors / editors / publishers of all those articles, journals and books from where the literature for this article has been reviewed and discussed.

\section{Conflict of interest: None}

\section{Source of Funding: Nil}

\section{REFERENCES}

1. Williams HP. Manipulation of the iris in extracapsular lens extraction. Trans Ophthalmol Soc UK 1985;104:553-7.

2. Goodman DF ,Stark WJ, Gottsch JD. Complications of cataract extraction with intraocular lens implantation. OphthalmolSurg 1989;20:132-40.

3. Akman A, Yilmaz G, Oto S, Akova YA. Comparison of various pupil dilatation methods for phacoemulsification in eyes with a small pupil secondary to pseudoexfoliation. Ophthalmology. 2004;111(9):1693-8.

4. Lowenstein O, Levine AS. Pupillographic studies. V. Periodic sympathetic spasm and relaxation and role of sympathetic nervous system in pupillary innervation.Arch Ophthalmol 1944;31:74.

5. Thompson HS. The pupil. In: Moses RA, Hart WM Jr,eds. Adler's physiology of the eye. St Louis: Mosby,1989:311-38.

6. Duffin RM, Canras CB, Gardner SK, Pettit TH. Inhibitors of surgically-induced miosis. Ophthalmology1982;89: 966-79.

7. Kongsap P. Visual outcome of manual small-incision cataract surgery: comparison of modified Blumenthal and Ruit techniques. Int J Ophthalmol, 2011;4(1):62-5.

8. Rim TH, Kim MH, Kim WC, Kim TI, Kim EK. Cataract subtype risk factors identified from the Korea National Health and nutrition examination survey 2008-2010. BMC Ophthalmol. 2014;14:4. 
9. Kumar, S., S. Jain, A. Wanjari, and S. Mandal. "Development and Validation of a Modified Frailty Risk Index as a Predictor of Mortality in Rural Elderly People." Asian Journal of Gerontology and Geriatrics 14, no. 1 (2019): 15-22.

10. Sharma U, Sharma B, Kumar K, Kumar S. Evaluation of complications and visual outcome in various nucleus delivery techniques of manual small incision cataract surgery. Indian J Ophthalmol. 2019;67(7):1073-1078.

11. Khokhar S, Pangtey MS, Soni A. Surgical peculiarities in type II diabetic cataracts during phacoemulsification. Ophthalmic Surg Lasers Imaging. 2003;34:100-103.

12. Zaczek A, Zetterström C. Cataract surgery and pupil size in patients with diabetes mellitus. Acta Ophthalmol Scand. 1997; 75:429-432.

13. Ostri C, Lund-Andersen H, Sander B, La Cour M. Phacoemulsification cataract surgery in a large cohort of diabetes patients: visual acuity outcomes and prognostic factors. J Cataract Refract Surg. 2011;37:2006-2012.

14. Zarnowski T, Machowicz-Matejko E, Zagórski Z. Anterior chamber inflammation following cataract surgery in patients with non-insulin-dependent diabetes mellitus. KlinOczna. 2002;104:354-356. Polish.

15. Phadnis, P., M.A. Kamble, S. Daigavane, P. Tidke, and S. Gautam. "Prevalence and Risk Factors - Hemoglobin A1c, Serum Magnesium, Lipids, and Microalbuminuria for Diabetic Retinopathy: A Rural Hospital-Based Study." Journal of Datta Meghe Institute of Medical Sciences University 12, no. 2 (2017): 121 32.

16. Joshi RS. Phacoemulsification without preoperative mydriasis in patients with age-related cataract associated with type 2 diabetes. Clin Ophthalmol. 2016;10:2427-2432.
17. Bozkurt E, Yazici AT, Pekel G, Albayrak S, Cakir M, Pekel E, et al. Effect of intracameral epinephrine use on macular thickness after uneventful phacoemulsification. J Cataract Refract Surg. 2010;36:1380-4.

18. Dohlman, C.H. and Hyndiuk, R.A. Subclinical and manifest corneal oedema after cataract ex- traction. Symposium on the cornea. Transactions of the New Orleans Academy of Ophthalmol- ogy. St Louis: Mosby, 1972, p. 221.

19. Hull, D.S., Chemotti, M.T., Edelhauser, HE., Van Horn, D.L. and Hyndiuk, R.A. Effect of adrenaline on the corneal endothelium. Am. J. Ophthalmol. 79(2):245-250, 1975.

20. Edelhauser HF, Hyndiuk RA, Zeeb A, et al. Corneal edema and the intraocular use of epinephrine. Am J Ophthalmol 1982;93:327-33.

21. Melanie C Corbett, Andrew B Richards, Intraocularadrenalinemaintainsmydriasisduring cataractsurgery,British Journal of Ophthalmology 1994;78:95-98

22. Liou, S.W., Yang, C.Y. The Effect of Intracameral Adrenaline Infusion on Pupil Size, Pulse Rate, and Blood Pressure During Phacoemulsification. Journal of Ocular Pharmacology and Therapeutics, 1998,14(4), 357-361.

23. Liou SW, Chen CC. Maintenance of mydriasis with one bolus of epinephrine injection during phacoemulsification. J Ocul Pharmacol Ther. 2001;17(3):249-53.

24. Farahat, Hassan Gamal El-Din; Ibrahim, Asmaa Muhammed and ALI, Ahmed Abdelwahab. The effect of intracameral epinephrine on pupil size during phacoemulsification and its postoperative effect on specular findings and macular thickness. Rev. bras. of talmol.[online]. 2016, vol.75, n.6 [cited 2020-04-15], pp.425-431. 